\title{
ORIGINAL ARTICLE High-dose ascorbic acid administration improves functional recovery in rats with spinal cord contusion injury
}

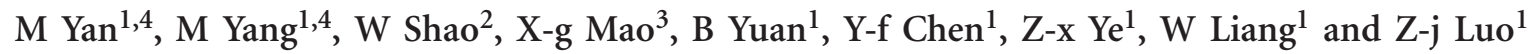

Objectives: To evaluate the effects of different doses of ascorbic acid $(A A)$ on the functional performance of rats subjected to standardized spinal cord injury (SCI).

Methods: Thirty female Sprague-Dawley rats were divided into three groups (10 animals in each group): control group: rats were subjected to $\mathrm{SCl}$ injury and received intraperitoneal saline administration; normal-dose AA group: rats were subjected to $\mathrm{SCl}$ injury and received daily intraperitoneal administration of $A A$ at $100 \mathrm{mg} \mathrm{kg}^{-1}$ bodyweight; high-dose $A A$ group: rats were subjected to SCl injury and received daily intraperitoneal administration of AA at $200 \mathrm{mg} \mathrm{kg}^{-1}$ bodyweight. The Basso, Beattie, Bresnahan Locomotor Rating Score (BBB score) and footprint analysis were performed to evaluate the functional performance of the rats in each group, and hematoxylin and eosin staining was performed to evaluate necrosis at the injury site.

Results: At days 14 and 28 after SCI, rats in the high-dose AA group, but not the normal-dose AA group, exhibited significantly better BBB score compared with the control group $(P<0.05)$. Compared with the control and normal-dose AA group, the high-dose AA group also showed increased stride length, decreased stride width and reduced toe dragging $(P<0.05)$. Histological analysis revealed that both the normal- and high-dose AA groups had reduced necrosis in the injury site compared with the control group ( $P<0.05)$.

Conclusion: High-dose AA administration during the acute phase post SCl significantly reduced secondary injury-induced tissue necrosis and improved functional performance in rats.

Spinal Cord (2014) 52, 803-808; doi:10.1038/sc.2014.135; published online 2 September 2014

\section{INTRODUCTION}

Spinal cord injury (SCI) results in severe neurological damage and requires extremely expensive, long-term care. ${ }^{1}$ Paraplegia and quadriplegia largely affect the quality of life of SCI patients, and has a major impact on their families and their relationship with society in general. SCI caused by trauma mainly occurs by two mechanisms: the primary injury and subsequent secondary injury, which itself can cause continuous damage to the function and structure of the spinal cord surrounding the primary injury region. ${ }^{2}$ Much effort has been made to understand the mechanism of secondary injury and devise therapeutic strategies for its inhibition. ${ }^{1}$ To date, there are very limited treatments available to prevent secondary injury and protect spinal cord functions after SCI. ${ }^{3}$ Important advances have been made in recent decades, with many therapeutic strategies being investigated, including stem cell transplantation. ${ }^{2}$ However, there are still controversies surrounding stem cell therapy and it is a complicated process in practice. ${ }^{2,4}$ Therefore, developing novel effective and convenient treatments for SCI is urgently required.

Although high dose of methylprednisolone administration remains controversial, ${ }^{3}$ it is the only proven treatment for acute SCI within $8 \mathrm{~h}$ post injury. ${ }^{5}$ The successful use of methylprednisolone treatment, although limited, gives hope for development of more effective drugs for SCI. Among the secondary injury events after SCI, free radicals, which are increased in the injury region after SCI, have an important role in causing progressive physiological and biochemical damage. ${ }^{6,7}$
The level of endogenous antioxidants, such as ubiquinols and ascorbate, decrease after SCI causing further oxidative damage and free radical accumulation. ${ }^{8}$ On the basis of these observations, several exogenous antioxidants capable of scavenging free radicals have been proposed as a treatment regimen for SCI, with some exhibiting beneficial effects, such as ascorbic acid (AA) $)^{9,10}$ and the nonglucocorticoid 21-aminosteroid tirilazad. ${ }^{11}$ Among these antioxidants agents, AA is of particular interest because of its critical function in a wide range of physiological processes. ${ }^{12}$ Several studies have investigated the therapeutic effect of AA administration on rat SCI models. ${ }^{9}$ A recent study suggested that AA did not improve neurological performance in the rat SCI model, but decreased the inflammatory response observed by histopathological examination. ${ }^{9}$ These results imply that AA has beneficial effects on SCI, and should be further explored for its clinical application in the treatment of SCI. Because of the profound change in the microenvironment and metabolic state after SCI, an excessive amount of beneficial agents may be needed to repair the lesion. For example, high-dose methylprednisolone may have beneficial effects for SCI in the acute phase. Previous results showing that AA only improved histopathological defects, but not neurological performance, may be owing to an insufficient dose of AA being used in the studies. Given the fact that few studies have been translated into clinical practice because of safety issues or uncertain outcomes, and that AA is widely used clinically with few side effects, it would be specifically important

\footnotetext{
${ }^{1}$ Department of Orthopaedic Surgery, Xijing Hospital, Fourth Military Medical University, Xi'an, China; ${ }^{2}$ Department of Orthopaedic Surgery, The 359 Hospital of Chinese PLA, Jiangsu, China and ${ }^{3}$ Department of Neurosurgery, Xijing Hospital, Fourth Military Medical University, Xi'an, China

${ }^{4}$ These authors contributed equally to the work.

Correspondence: Professor Z-j Luo, Department of Orthopaedic Surgery, Xijing Hospital, Fourth Military Medical University, No 127 Changle Road, Xi'an 710032 , China. Email: zjluo@fmmu.edu.cn

Received 28 February 2014; revised 20 June 2014; accepted 12 July 2014; published online 2 September 2014
} 
to further explore whether AA has beneficial effects for SCI. Therefore, the aim of this study was to systematically evaluate the effects of high-dose AA on SCI rats.

\section{MATERIALS AND METHODS}

\section{Animals}

Thirty female Sprague-Dawley rats (200-220 g, provided by the Animal Center of the Fourth Military Medical University) were randomly divided into three groups. (1) control group: SCI rats receiving saline intraperitoneally (i.p.); (2) normal-dose AA group: SCI rats receiving AA of $100 \mathrm{mg} \mathrm{kg}^{-1}$ per day i.p. ; $^{9}$ (3) high-dose AA group: SCI rats receiving AA $200 \mathrm{mg} \mathrm{kg}^{-1}$ per day i.p. All of the rats were maintained on a $12 / 12 \mathrm{~h}$ light/dark cycle and were allowed free access to food and water. The procedures were reviewed and approved by the Animal Care Committee of the Fourth Military Medical University. All efforts were made to minimize the number of animals used and their suffering. At the end of the studies, all rats were euthanized with an overdose of sodium pentobarbital.

\section{Surgical procedure}

The rats were anesthetized with $1 \%$ sodium pentobarbital $\left(50 \mathrm{mg} \mathrm{kg}^{-1}\right)$ i.p. A 30-40-mm dorsal midline incision was made at the T8-T12 level, and bilateral laminectomy was performed to expose the vertebrate T10 spinal cord. The NYU Impactor (MASCIS, New York University, New York, NY, USA) was used to produce a contusion SCI and to monitor the contusion. ${ }^{13}$ After being placed in the Impactor frame, the $\mathrm{T} 8$ and $\mathrm{T} 11$ processes were clamped, and the Impactor rod was adjusted to the zero position, after which the basal clip was attached to the border of the surgical wound. The Impactor was then lowered between the upper margin of T9 and the lower margin of T10. After contacting with the spinal cord, as indicated by means of audible and visible signals of the device caused by closing the circuit between the basal clip and the Impactor head, the Impactor rod was raised to a height of $25 \mathrm{~mm}$. The height and date information were entered into the Impactor program. The Impactor rod was then released to hit the spinal cord, which was lifted off immediately after producing the contusion. The contusion site was inspected, active bleeding was stopped and the paravertebral muscles and the skin were closed. Then the operated animals were kept in cages with soft bedding and ambient temperature maintained at $20-23^{\circ} \mathrm{C}$.

\section{Drug administration}

An hour after the injury, the rats received i.p. administration of saline (control group), $100 \mathrm{mg} \mathrm{kg}^{-1}$ bodyweight per day of AA (normal-dose AA group), or $200 \mathrm{mg} \mathrm{kg}^{-1}$ bodyweight per day of AA (high-dose AA group). Over the days following the injury, the rats received daily doses of the same vitamins, until the day they were killed. All rats received postoperative treatment including the intraperitoneal injection of penicillin for a week. Bladders were manually voided three times daily until reflex bladder emptying was established. The rats were maintained for 4 weeks under postoperative care recommended by the National Institutes Of Health Guide for Care and Use of Laboratory Animals, and were given access to food and water ad libitum.

\section{Functional assessment}

The BBB Locomotor Rating Scale was used to evaluate the recovery of hindlimb locomotor functions in an open field. ${ }^{14}$ The behavioral evaluations were carried out at $0800-0900$ hours weekly for 4 weeks after operation. The BBB locomotor rating scale ranges from 0 , which corresponds to flaccid paralysis, to 21 , which is normal gait. Rats were allowed to walk around freely in a spacious field for $5 \mathrm{~min}$ when the movement of the hindlimbs was closely observed. Three observers blind to the animals' identity assisted in this task. The average of the three scores of the three observers was calculated. Before testing, bladders were expressed, as spontaneous bladder contraction often accompanied hindlimb activity.

\section{Footprint analysis}

The analysis was conducted weekly for 4 weeks after operation. The plantar surface of both hindlimbs of each rat was colored black and the dorsal surface red with nontoxic inks. The rat was then allowed to run toward a dark tunnel on the white paper $\left(21 \times 59.4 \mathrm{~cm}^{2}\right)$, thus the red and/or black ink was printed onto the paper as one set of footprints. ${ }^{15}$ From each set of footprints, the pixels of the black (normal plantar paw placing) and red (abnormal dorsal paw placing) areas were determined using ImageJ (http://rsb.info.nih.gov/ij/). The percentage of red pixels defined as red/(black + red $) \times 100 \%$ was computed to quantify toe dragging. Stride length (distance between the centers of ipsilateral adjacent footprints) and stride width (perpendicular distance between the centers of left and right hindlimbs) were measured, and the average of five steps in each case was used for statistical comparison.

\section{Histological investigation of spinal cord}

At 4 weeks after SCI, the rats were anaesthetized with intraperitoneal injection of $1 \%$ sodium pentobarbital $\left(50 \mathrm{mg} \mathrm{kg}^{-1}\right)$ as described above, and 11 of $0.1 \mathrm{mmoll}^{-1}$ phosphate-buffered saline were introduced into the left ventricle for $20-30 \mathrm{~min}$, followed by 11 of $4 \%$ paraformaldehyde in $0.1 \mathrm{moll}^{-1}$ phosphate-buffered saline ( $\mathrm{pH} 7.2-7.3,4^{\circ} \mathrm{C}$ for $1 \mathrm{~h}$ ). The spinal cords were taken out and immersed in the same fixative at $4{ }^{\circ} \mathrm{C}$ for $24 \mathrm{~h}$, then transferred to phosphate buffer containing $30 \%$ sucrose before cryosectioning. Serial sections of the spinal cord tissues were cut at a thickness of $20 \mu \mathrm{m}$ (some sagitally and others coronally) in a cryo-microtome (Leica CM 1900, Leica Microsystems Nussloch GmbH, Nussloch, Germany) and mounted on gelatinpretreated slides for the following laboratory procedures. Hematoxylin and eosin staining was performed for histological analysis.

\section{Quantification of area of injury}

Hematoxylin and eosin-stained sections were used to evaluate the necrosis area in the injured site. Five sections were chosen for analysis in each rat, namely, the section with central canal and two adjacent sections on both sides, with $200 \mu \mathrm{m}$ between each section. The microscopic images were obtained with a $\times 40$ objective under an Olympus BX-51 microscope (Olympus Corporation, Tokyo, Japan).

\section{Statistical analysis}

All data were expressed as mean \pm s.d. Statistical analysis were performed using Student's $t$-tests and one-way analysis of variance with least squared difference post hoc tests, as appropriate. All $P$-values are two tailed. A value of $P<0.05$ was considered statistically significant. Statistical analyses were done using SPSS v.13.0.0 (SPSS Inc., Chicago, IL, USA).

\section{RESULTS}

\section{BBB scoring}

$\mathrm{BBB}$ scores were examined to evaluate the effects of different doses of $\mathrm{AA}$ on the overall function of SCI rats. The BBB score of high- and normal-dose AA groups showed gradual increased benefits compared with the control group after SCI (Figure 1). The BBB score of the high-dose AA group was significantly higher than that of the normaldose AA and control groups at 14 days $(10.50 \pm 1.08$ vs $8.30 \pm 1.06$ and 7.10 $\pm 0.86, P<0.05$, Figure 1 ; Table 1$)$ and 28 days (15.80 \pm 1.81 vs $13.60 \pm 1.51$ and $11.20 \pm 2.04, P<0.05$, Figure 1; Table 1) after SCI. Consistent with previous reports, the normal-dose AA group showed higher $\mathrm{BBB}$ scores than the control group, but they were not statistically significant (Figure 1). Noticeably, the functional recovery of hindlimbs in the high-dose AA group improved more apparently than the normal-dose AA and control groups, as indicated by the observation that some rats in the high-dose AA group showed large movements of the third joint of the hindlimb (see detail in footprint analysis).

\section{Footprint analysis}

Toe dragging, stride length and stride width were further analyzed to evaluate the functional recovery of rats in each group (Figure 2). As shown in Figure 2, normal rats showed consistent plantar stepping 
during the whole process (Figure 2a), whereas severe toe dragging with no planar stepping was observed in the control group at 28 days after SCI (Figure 2b). After SCI, the stride length increased, and the stride width decreased progressively. However, the high- and normaldose AA groups exhibited greater increased stride length, deceased stride width and less toe dragging compared with the control group (Figures 2-4; Tables 2 and 3).

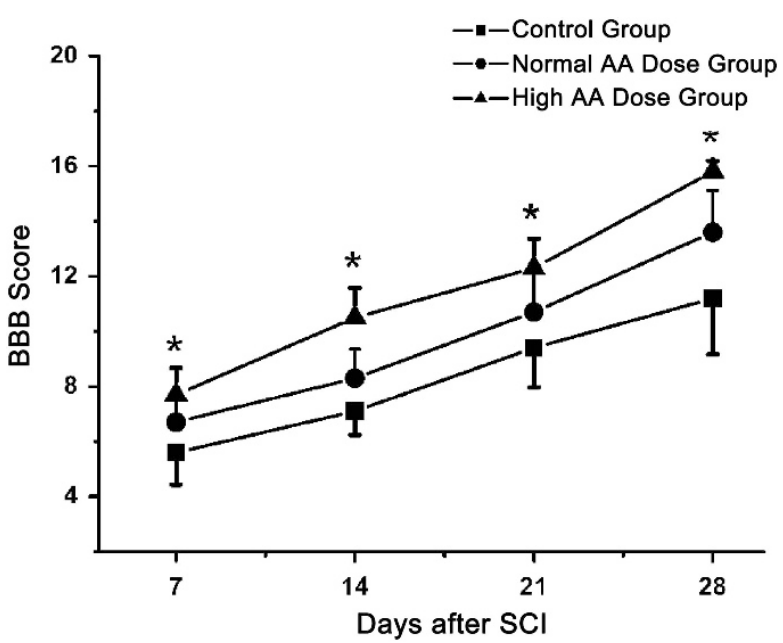

Figure 1 Graph showing the BBB score of each group at different time points after $\mathrm{SCl} .{ }^{*} P<0.05$.

Table 1 The Basso, Beattie, Bresnahan Locomotor Rating Score (BBB score) of each group after $\mathrm{SCl}$ at different time points (mean \pm s.d., $n=10$ )

\begin{tabular}{lcccc}
\hline Groups & $7 d$ & $14 d$ & $21 d$ & $28 d$ \\
\hline Control & $5.60 \pm 1.17$ & $7.10 \pm 0.86$ & $9.40 \pm 1.43$ & $11.20 \pm 2.04$ \\
Normal-dose AA & $6.70 \pm 0.95^{\ddagger}$ & $8.30 \pm 1.06^{\ddagger}$ & $10.70 \pm 1.57^{\ddagger}$ & $13.60 \pm 1.51^{\ddagger}$ \\
High-dose AA & $7.70 \pm 0.96^{*}$ & $10.50 \pm 1.08^{*}$ & $12.30 \pm 1.06^{*}$ & $15.80 \pm 1.81^{\#}$ \\
\hline
\end{tabular}

Abbreviations: $\mathrm{AA}$, ascorbic acid; d, days; $\mathrm{SCl}$, spinal cord injury.

Note: ${ }^{*} P<0.05,{ }^{\#} P<0.01$ and $\ddagger P>0.05$ compared with the control group.
Quantitative analysis demonstrated that the high-dose AA group exhibited increased stride length compared with both the control and normal-dose AA groups at 14 days $(11.80 \pm 1.32 \mathrm{~cm}$ vs $10.24 \pm 1.02$ and $10.11 \pm 0.95 \mathrm{~cm} ; P<0.05$, Figure 3a; Table 2) and 28 days $(12.78 \pm 0.99 \mathrm{~cm}$ vs $11.20 \pm 1.16$ and $10.58 \pm 0.99 \mathrm{~cm} ; \quad P<0.05$,
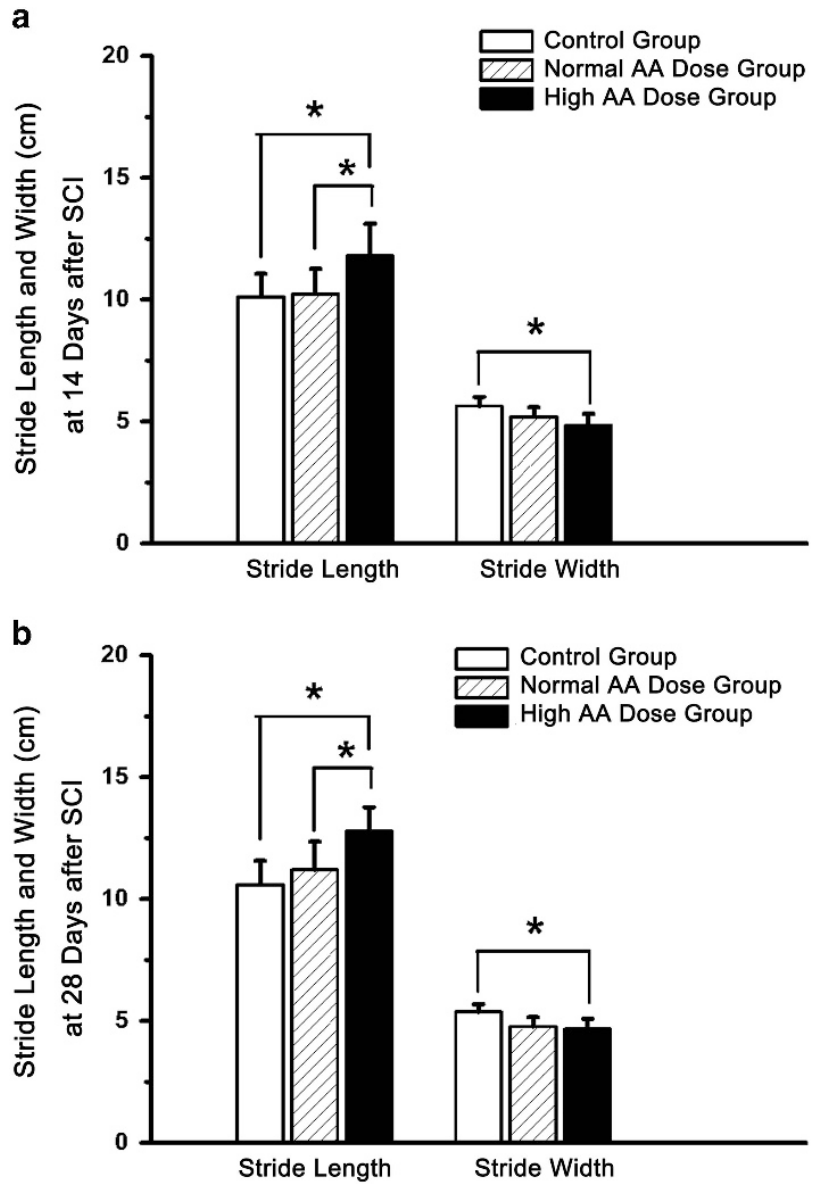

Figure 3 Graph showing the quantitative results of footprint analysis (stride length and stride width) for each group at 14 (a) and 28 (b) days after $\mathrm{SCl}$. ${ }^{*} P<0.05$.

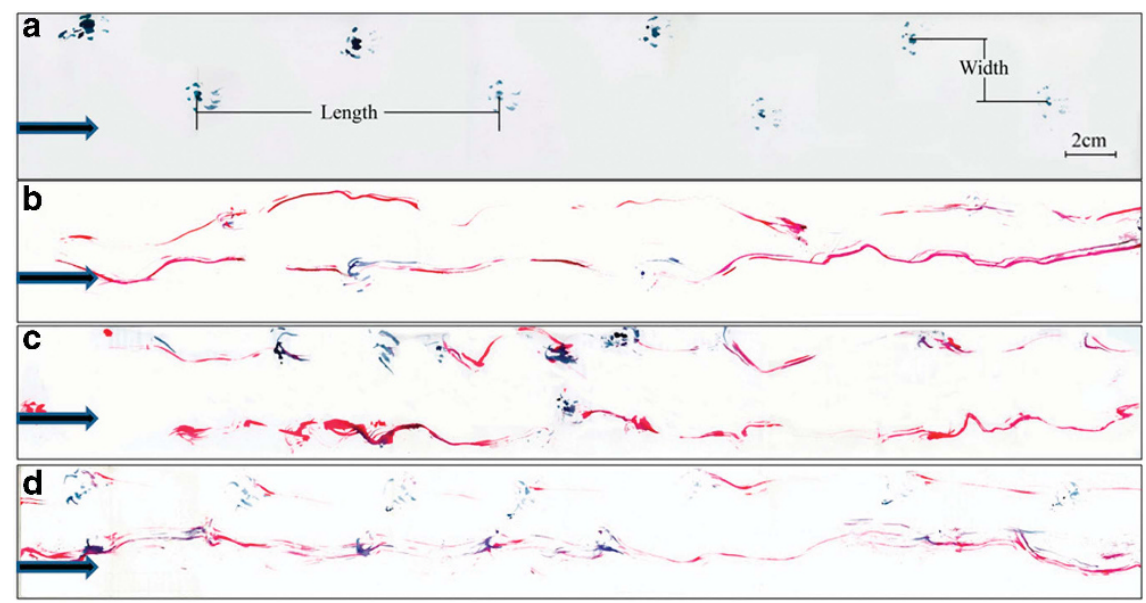

Figure 2 Representative images showing the footprint for each group at 14 days after SCl. (a) Normal rat; (b) control group; (c) normal-dose AA group; (d) high-dose AA group. 
Figure 3b; Table 3) after SCI. In addition, the high-dose AA group also exhibited a significant decrease in stride width compared with the control group at 14 days $(4.85 \pm 0.46 \mathrm{~cm}$ vs $5.63 \pm 0.38 \mathrm{~cm}, P<0.05$, Figure 3a; Table 2) and 28 days $(4.66 \pm 0.42 \mathrm{~cm}$ vs $5.37 \pm 0.31 \mathrm{~cm}$, $P<0.05$, Figure 3b; Table 3) after SCI. The stride width of the high-dose AA group was also less than that of the normal-dose AA group, although it was not statistically significant (Figures $3 \mathrm{a}$ and $\mathrm{b}$; Tables 2 and 3). Noticeably, at 28 days after SCI, the normal-dose AA group showed increased stride length compared with the control group ( $4.77 \pm 0.38 \mathrm{~cm}$ vs $5.37 \pm 0.31 \mathrm{~cm}, P<0.05$, Figure $3 \mathrm{~b}$; Table 3 ), indicating that normal AA dose also has a significant benefit for functional recovery of SCI rats at later time points.

Further analysis of toe dragging revealed that the ratio of rats with toe dragging in the high-dose AA group was $35.11 \pm 1.47 \%$ and

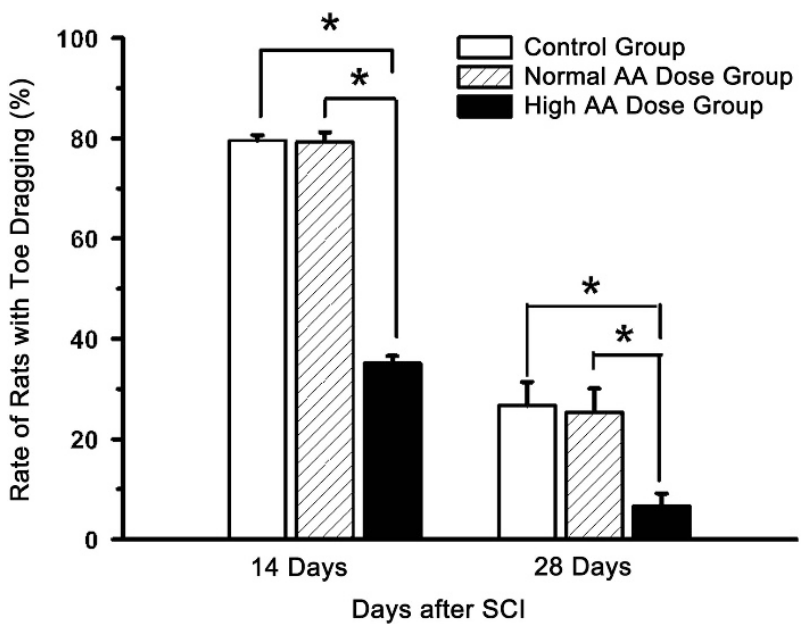

Figure 4 Graph showing the quantitative results of toe dragging ratio of each group at 14 and 28 days after $\mathrm{SCl}$. ${ }^{*} P<0.05$.

Table 2 The stride length, stride width and toe dragging for each group at 14 days after $\mathrm{SCl}$ (mean \pm s.d., $n=10$ )

\begin{tabular}{llll}
\hline Groups & Stride length $(\mathrm{cm})$ & Stride width $(\mathrm{cm})$ & $\begin{array}{l}\text { Ratio of rats with } \\
\text { toe dragging (\%) }\end{array}$ \\
\hline Control & $10.11 \pm 0.95$ & $5.63 \pm 0.38$ & $79.57 \pm 1.11$ \\
Normal-dose AA & $10.24 \pm 1.02$ & $5.19 \pm 0.39$ & $79.29 \pm 1.95$ \\
High-dose AA & $11.80 \pm 1.32^{*, \dagger}$ & $4.85 \pm 0.46^{*}$ & $35.11 \pm 1.47^{*, \dagger}$ \\
\hline
\end{tabular}

Abbreviations: $\mathrm{AA}$, ascorbic acid; $\mathrm{SCl}$, spinal cord injury.

Note: ${ }^{*} P<0.05$, ${ }^{\#} P<0.01$ compared with the control group; ${ }^{\dagger} P<0.01$ compared with the normal-dose AA group.

Table 3 The stride length, stride width and toe dragging for each group at 28 days after $\mathrm{SCl}$ (mean \pm s.d., $n=10$ )

\begin{tabular}{lllc}
\hline Groups & Stride length $(\mathrm{cm})$ & Stride width $(\mathrm{cm})$ & $\begin{array}{c}\text { Ratio of rats with } \\
\text { toe dragging (\%) }\end{array}$ \\
\hline Control & $10.58 \pm 0.99$ & $5.37 \pm 0.31$ & $26.67 \pm 4.73$ \\
Normal-dose AA & $11.20 \pm 1.16$ & $4.77 \pm 0.38^{*}$ & $25.32 \pm 4.74$ \\
High-dose AA & $12.78 \pm 0.99^{*, \dagger}$ & $4.66 \pm 0.42^{*}$ & $6.61 \pm 2.57^{*, \dagger}$
\end{tabular}

Abbreviations: AA, ascorbic acid; $\mathrm{SCl}$, spinal cord injury.

Note: ${ }^{*} P<0.05,{ }^{\#} P<0.01$ compared with the control group; ${ }^{\dagger} P<0.01$ compared with the normal-dose AA group.
$6.61 \pm 2.57 \%$ at 14 and 28 days after SCI, respectively, which is significantly lower than that of the control group $(79.57 \pm 1.11 \%$ and $26.67 \pm 4.73 \%$ at 14 and 28 days, respectively; $P<0.05$, Figure 4 ; Tables 2 and 3$)$ and normal-dose AA group $(79.29 \pm 1.95 \%$ and $25.32 \pm 4.74 \%$ at 14 and 28 days, respectively; $P<0.05$, Figure 4; Tables 2 and 3). Notably, the normal-dose AA group did not have an apparent reduced toe-dragging rate compared with the control group (Figure 4; Tables 2 and 3).
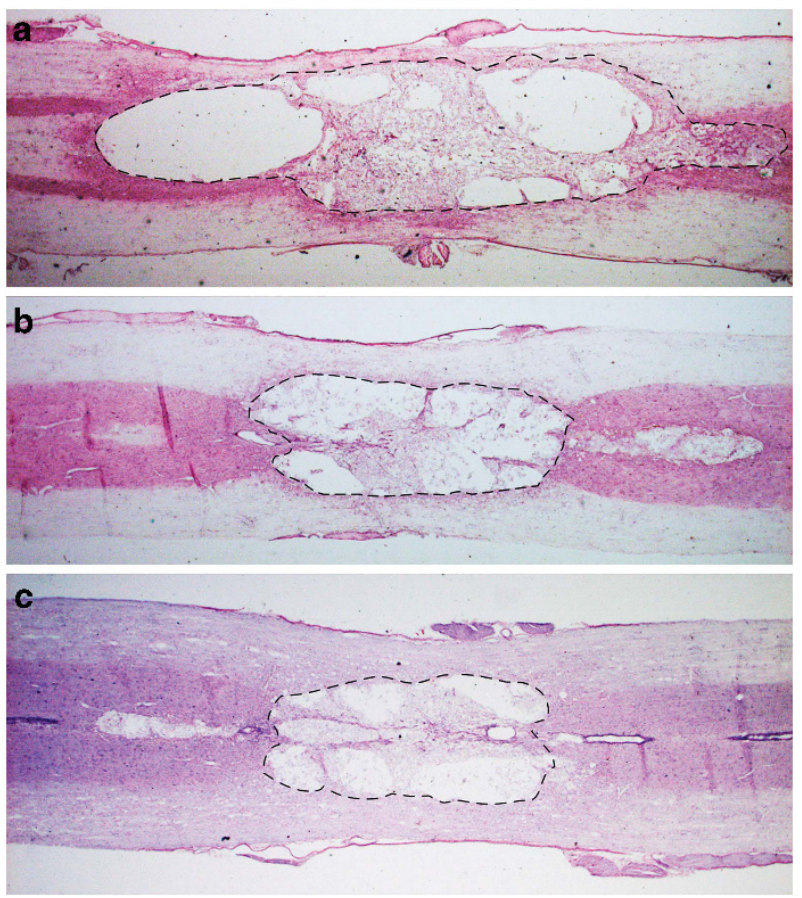

Figure 5 Representative images showing the lesion area at the section with central canal in each group at 28 days after $\mathrm{SCl}$. The lesion area is outlined with dashed lines. (a) Control group; (b) normal-dose AA group; (c) highdose AA group.

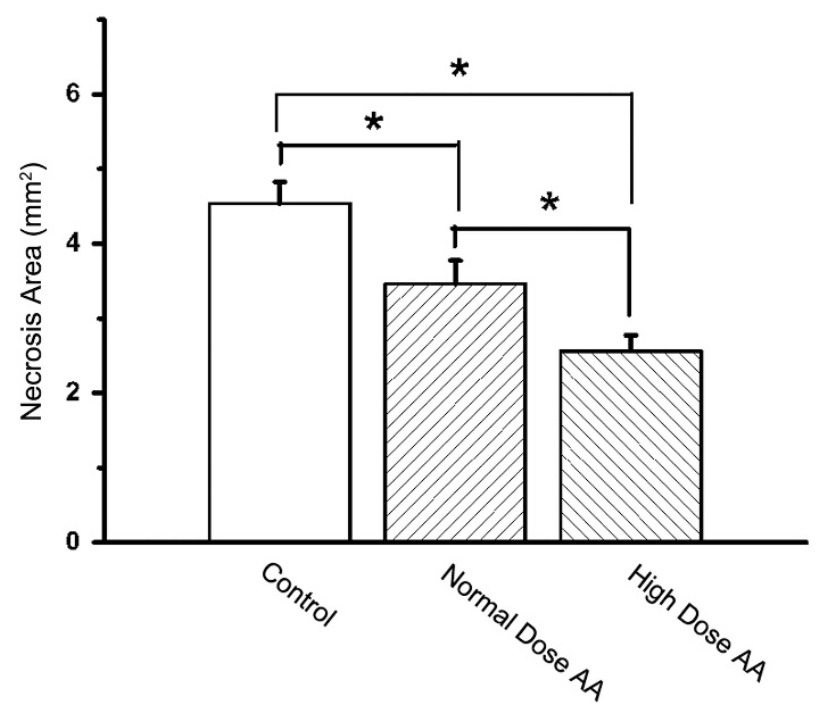

Figure 6 Graph showing the quantitative results of the lesion area in each group. ${ }^{*} P<0.05$. 


\section{Histology}

We next analyzed the histological changes of the injured spinal cord for each group. The rats were euthanized at 28 days after SCI, and the spinal cords were sagittally sectioned and stained with hematoxylin and eosin stain. Extensive necrosis and prominent cavitation was observed at the injury site in the control group (Figure 5a), whereas the necrosis and cavitation area were much smaller in the normaland high-dose AA groups (Figures $5 \mathrm{~b}$ and c). Quantitative analysis revealed that the lesion area (including the necrosis and cavitation area) in the high-dose AA group $\left(2.57 \pm 0.20 \mathrm{~cm}^{2}\right)$ was much smaller than that of the control group $\left(4.55 \pm 0.28 \mathrm{~cm}^{2}, P<0.05\right.$, Figure 6) and the normal-dose AA group $\left(3.48 \pm 0.31 \mathrm{~cm}^{2}, P<0.05\right.$, Figure 6). Consistent with previous reports, the lesion area in the normal-dose AA group was also significantly smaller than that of the control group $(P<0.05$, Figure 6)

\section{DISCUSSION}

After the primary insults of SCI, secondary injury was triggered by a rapid inflammatory response. ${ }^{16}$ Many processes contribute to secondary injury, including lipid peroxidation, free radical production, inflammation, ischemia, glutamate excitotoxicity, cytoskeletal degradation and the induction of extrinsic and intrinsic apoptotic pathways. ${ }^{17}$ Free radicals and proinflammatory cytokines can cause a series of effects leading to progressive damage after SCI. ${ }^{6,11}$ Free radicals not only attack and damage the membrane of neural cells, but also destroy the critical enzymatic systems in the phospholipid membrane, such as the activity of the $\mathrm{Na}^{+}-\mathrm{K}^{+}$-ATPase, resulting in swelling and dysfunction of neural cells, and ultimately their autolysis. ${ }^{6}$ Physiologically, endogenous catalase and superoxide dismutase have functions that help clear free radicals. However, excess free radicals are produced after SCI by many mechanisms, such as hemorrhage, inflammatory cell phagolysis and ischemia-induced energy dysmetabolism. The excess free radicals, which cannot be removed by endogenous enzymes, have important roles in the secondary injury process. Therefore, the clearance of these excessive free radicals after SCI is an important strategy to reduce injury and protect neural cells.

The infiltration of immune cells into the injury site of the SCI contributes significantly to secondary degeneration. ${ }^{17}$ The inflammatory response after SCI is particularly important, because post-traumatic inflammation is larger in the spinal cord than other areas in the central nervous system, such as the brain. After SCI, neutrophils, activated macrophages and microglia infiltrate into the injury site and can persist there for several months. Although the inflammatory response mediated by these cells is required for efficient clearance of tissue debris, and promotes tissue repair, they also release various factors that are detrimental to neurons, glia, axons and myelin. For example, proinflammatory cytokines upregulated after SCI induce other biochemical cascades leading to the degeneration of myelin and neuronal apoptosis. ${ }^{18}$ Therefore, components of the central nervous system inflammatory response have been suggested to be important targets to reduce secondary injury after SCI.

It has been recognized that a small increase in the proportion of axons protected after SCI could have great impact on behavioral and functional consequences leading to markedly improved quality of life for SCI patients. Therefore, given the fact that neural damage caused by the initial insults is largely irreversible, it could be more important to protect the remaining neurons and axons that are subsequently damaged by secondary injury.
As one type of indispensable nutritive factor, AA has multiple important functions in the human body. ${ }^{10,12,19}$ One of the vital functions of $\mathrm{AA}$ is to clear free radicals, therefore exerting protective effect in various injuries. ${ }^{12}$ Therefore, AA has been used for the treatment of many diseases, such as cardiovascular, digestive, respiratory, musclar, immune dysfunction, urogenital and, importantly, neurological diseases. ${ }^{10,20}$ In fact, the effect of normaldose AA on SCI was previously investigated, and demonstrated that AA can alleviate the histological features of tissue injury, although it did not show significant effect on the behavioral function of the injured animals. ${ }^{9}$ The results suggest that AA has positive effects on SCI; however, its usage at a normal dose has only limited protective effects. Interestingly, it has been illustrated for certain neurological diseases that only high doses of AA have therapeutic effects, implying that a higher dose of AA might have additional mechanisms to have protective roles in the nervous system.

Herein, we evaluated the protective effects of normal and high doses of AA in the rat SCI model. Dorsal contusion injury to the rat spinal cord was induced with Impactor Model I system, and the recovery of the rats after SCI was evaluated histologically and behaviorally. Histologically, both normal- and high-dose AA reduced the necrotic area at the injury site compared with the control group $(P<0.05)$. These results confirmed the protective effects of AA, ${ }^{9}$ and further demonstrated that high-dose AA has better therapeutic effects as it reduced the necrotic area significantly compared with the normal dose. Interestingly, the behavioral test revealed that the BBB score of the high-dose AA group was significantly better than that of normaldose AA and control groups at each time point, whereas there was no statistically significant difference between the normal-dose AA and control groups. In addition, footprint analysis revealed that the stride length and stride width in the high-dose AA group, but not the normal-dose AA group, was significantly higher than those in the control group. The high-dose AA group also showed significantly longer stride length than the normal-dose AA group. These results imply that administration of high-dose AA in the early stages after SCI significantly reduced the secondary injury and improved functional recovery.

Our work demonstrates that both normal-dose and high-dose AA reduced the secondary injury after SCI by histological examination, however, only the high-dose AA improved behavioral function significantly. Although normal-dose AA reduced the necrotic area, the degree of tissue recovery rescued may not sufficient to significantly improve behavioral function. In addition, improved behavioral function of the rats required not only the recovery of damaged neural tissue but also the improved motion of joints, muscle strength and coordination of the limbs. Given the multiple roles of AA, especially its protective roles for muscle loss and damage, ${ }^{19}$ immune dysfunction and peripheral nervous system and cardiovascular damage, the improved behavioral function of SCI rats following high-dose AA administration may be combination of these effects and the reduced necrosis at the injury site. Nevertheless, there might be additional mechanisms that contribute to the improved behavioral function that require further investigation.

In summary, high-dose AA administration in the acute phase post SCI significantly reduced the secondary damage-induced tissue necrosis and improved the behavioral function of the rats. To date, few studies have successfully translated into clinical practice owing to safety limitations or uncertain outcomes. Because AA is widely used in clinical practice with few side effects, is of low cost and is convenient to use, our results provide novel feasible therapeutic strategies for acute SCI treatment. Nevertheless, some issues should be further explored in 
future studies before clinical administration of AA after SCI. For example, the optimal time period after SCI for treatment with AA should be determined to obtain better therapeutic effects.

\section{DATA ARCHIVING}

There were no data to deposit.

\section{CONFLICT OF INTEREST}

The authors declare no conflict of interest.

\section{ACKNOWLEDGEMENTS}

This study was partially supported by the National Natural Science Foundation of China (81301581)

1 Thuret S, Moon LD, Gage FH. Therapeutic interventions after spinal cord injury. Nat Rev Neurosci 2006; 7: 628-643.

2 Silva NA, Sousa N, Reis RL, Salgado AJ. From basics to clinical: a comprehensive review on spinal cord injury. Prog Neurobiol 2014; 114: 25-57.

3 Bydon M, Lin J, Macki M, Gokalsan ZL, Bydon A. The current role of steroids in acute spinal cord injury. World Neurosurg (e-pub ahead of print 20 February 2013; doi:10.1016/j.wneu.2013.02.062).

4 Hewson SM, Fehlings LN, Messih M, Fehlings MG. The challenges of translating stem cells for spinal cord injury and related disorders: what are the barriers and opportunities? Expert Rev Neurother 2013; 13: 143-150.

5 Bracken MB, Shepard MJ, Holford TR, Leo-Summers L, Aldrich EF, Fazl M et al. Methylprednisolone or tirilazad mesylate administration after acute spinal cord injury: 1-year follow up. Results of the third National Acute Spinal Cord Injury randomized controlled trial. J Neurosurg 1998; 89: 699-706.
6 Halliwell B, Gutteridge JMC. Free Radicals in Biology and Medicine. Oxford University Press, 2007, p 162.

7 Silver J, Miller JH. Regeneration beyond the glial scar. Nat Rev Neurosci 2004; 5 $146-156$.

8 Hausmann ON. Post-traumatic inflammation following spinal cord injury. Spinal Cord 2003; 41: 369-378.

9 Cristante AF, Barros Filho TE, Oliveira RP, Marcon RM, Rocha ID, Hanania FR et al. Antioxidative therapy in contusion spinal cord injury. Spinal Cord 2009; 47 458-463.

10 Chen HC, Hsu PW, Tzaan WC, Lee AW. Effects of the combined administration of vitamins $C$ and $E$ on the oxidative stress status and programmed cell death pathways after experimental spinal cord injury. Spinal Cord 2013; 52: 24-28.

11 Hall ED. Antioxidant therapies for acute spinal cord injury. Neurotherapeutics 2011; 8: 152-167.

12 Guerra-Araiza C, Alvarez-Mejia AL, Sanchez-Torres S, Farfan-Garcia E, MondragonLozano R, Pinto-Almazan $\mathrm{R}$ et al. Effect of natural exogenous antioxidants on aging and on neurodegenerative diseases. Free Radic Res 2013; 47: 451-462.

13 Young W. Spinal cord contusion models. Prog Brain Res 2002; 137: 231-255.

14 Basso DM, Beattie MS, Bresnahan JC. A sensitive and reliable locomotor rating scale for open field testing in rats. J Neurotrauma 1995; 12: 1-21.

15 Saadoun S, Bell BA, Verkman AS, Papadopoulos MC. Greatly improved neurological outcome after spinal cord compression injury in AQP4-deficient mice. Brain 2008; 131: 1087-1098.

16 Varma AK, Das A, Gt Wallace, Barry J, Vertegel AA, Ray SK et al. Spinal cord injury: a review of current therapy, future treatments, and basic science frontiers. Neurochem Res 2013; 38: 895-905.

17 Zhang N, Yin Y, Xu SJ, Wu YP, Chen WS. Inflammation \& apoptosis in spinal cord injury. Indian J Med Res 2012; 135: 287-296.

18 Sahin B, Albayrak BS, Ismailoglu O, Gorgulu A. The effects of medroxy progesterone acetate on the pro-inflammatory cytokines, TNF-alpha and IL-1beta in the early phase of the spinal cord injury. Neurol Res 2011; 33: 63-67.

19 Welch AA. Nutritional influences on age-related skeletal muscle loss. Proc Nutr Soc 2014; 73: 16-33

$20 \mathrm{Heo} \mathrm{JH}$, Hyon L, Lee KM. The possible role of antioxidant vitamin C in Alzheimer's disease treatment and prevention. Am J Alzheimers Dis Other Demen 2013; 28: 120-125. 